\title{
Chaotification in the stretch-twist-fold (STF) flow
}

\author{
YUE BaoZeng $^{1} \&$ AQEEL Muhammad ${ }^{1,2^{*}}$ \\ ${ }^{1}$ Department of Mechanics, School of Aerospace Engineering, Beijing Institute of Technology, Beijing 100081, China; \\ ${ }^{2}$ Department of Mathematics, COMSATS Institute of Information Technology, Islamabad 44000, Pakistan
}

Received May 14, 2012; accepted August 8, 2012

\begin{abstract}
Chaotic behavior and detailed parameter analysis of stretch-twist-fold (STF) flow are investigated. STF flow is associated with fluid particle motion which naturally arises in the dynamo theory. It proposes a mechanism, by which a celestial bodies, such as earth and sun, can maintain and amplify the magnetic field continuously. Parameter analysis is performed using linearization theory for different choices of parameters. The existence of Heteroclinic trajectory of Sil'nikov type is proved using an undetermined coefficient method. It connects two non trivial equilibrium points. As a consequence, the Sil'nikov criterion guarantees that STF flow has Smale horseshoes chaos.
\end{abstract}

stretch-twist-fold flow, parameter analysis, undetermined coefficient method, heteroclinic orbit, Smale horseshoes chaos

Citation: $\quad$ Yue B Z, Aqeel M. Chaotification in the stretch-twist-fold (STF) flow. Chin Sci Bull, 2013, 58: 1655-1662, doi: 10.1007/s11434-013-5754-x

During last few decades, existence of chaos in nonlinear dynamical system became an attractive field for researchers $[1,2]$. In most of the technological fields, chaos has an immense prospective use, for example in signal processing, information and computer science, biomedical system analysis and flow dynamics [3-5]. STF flow is defined in the unit sphere which is a two parameter family of incompressible steady Stokes' flow. This flow represents mechanism of stretch, twist and fold which is prototype of a fast dynamo action that is, in astrophysics, natural growth of magnetic field caused by the motion of electrically conducting fluid. Vainshtein et al. [6] introduced STF mechanism for the first time in physical manners rather than mathematical, which is the example of fast dynamo action. 'Fast dynamo action' or STF action is shown in Figure 1. If we apply similar approach on magnetic flux tube in a conducting fluid and repeat the process again and again, the repetition of stretch, twist and fold mechanism results in an exponential growth of magnetic field [6]. On the conjecture of Vainshtein et al. [6], researchers invented a most conductive particular quadratic flow in the magneto hydrodynamics which exhibits the stretch, twist and fold mechanism $[7,8]$ and modified

\footnotetext{
*Corresponding author (email: medraqeel@gmail.com)
}

by Bajer [9]. It is observed that a rich chaotic Lagrangian structure is present in a class of three dimensional incompressible steady STF flows [10]. These flows incorporated the stretch-twist-fold fast dynamo process and for this reason these are called stretch-twist-fold flows. Earth is not the only body which manifests magnetic activity but such phenomenon also exists on the other stars. Rotation of the earth produces a Coriolis effects which rotate the liquid iron in the outer core of earth. The liquid iron in the outer core of the earth induces the constant magnetic field. The results obtained from the STF flow are useful to explore the magnetic field structure [8]. Because of the spontaneous growth of terrestrial space magnetic field, researchers are interested to explore novel techniques and tools for non fuel consumption magnetism propulsion for low earth orbit spacecrafts, such as Lorenz force method [11], solar sailing [12], photon propulsion [13] and electrodynamics tethers [14]. Magnetism propulsion gets effectively thrust force by producing static magnetic field and interaction with the terrestrial space magnetic field which is produced by the fast dynamo action.

Over the past few decades three-dimensional nonlinear quadratic systems gained much attention in physics, mathematics, and engineering communities [15]. In studying the 
non linear dynamical system, researchers try to find the complex and long term behavior solution for the differential equation. Since the pioneering work of Lorenz [16], many other simple chaotic systems have been discovered such as Lu system [17] and Chen system [18]. Bao and Yang [19] developed a novel technique for the determination of homoclinic and heteroclinic orbits. Undetermined coefficient method is developed by Zhou et al. [20]. It is an analytic technique, which is used for the existence of heteroclinic or homoclinic orbits of the Sil'nikov type in the dynamical systems. The dynamical system will exhibit Smale horseshoes chaos if Sil'nikov criterion is validated. This technique is used by many researchers [21-24]. Some basic and complex properties of the STF flow are analyzed by Bao and Yang [25]. We present a detailed analysis of local behavior of the equilibrium points for the different choices of parameters of the STF flow in this work. Existence of Sil'nikov chaos in STF flow is proved by novel technique of undetermined coefficient method. Sil'nikov criterion [24,26] gives a surety for existence of Smale horseshoes chaos in STF flow for the typical values of the parameters. It is proved that this system contains heteroclinic orbit which joins the two different saddle foci and satisfies all conditions of the Sil'nikov theorem [24,26]. It is the criteria for existence of Smale horseshoes chaos.

\section{Sil'nikov Theorem}

Consider the third order autonomous system:

$$
\frac{\mathrm{d} x}{\mathrm{~d} t}=f(x), \quad x \in R^{3}, t \in R,
$$

where the vector field $f(x): R^{3} \rightarrow R^{3} \in C^{r}(r \geqslant 2)$.

For the existence of chaos, we summarize the heteroclinic Sil'nikov method which is known as Sil'nikov criterion.

Theorem (The heteroclinic Sil'nikov Theorem [24,26]). For the third order autonomous system (1), consider that there are two different equilibrium points designated by $x^{1}$ and $x^{2}$, which are assumed to be saddle foci having eigenvalues $\delta_{k}$, and $\sigma_{k} \pm i \omega_{k}$ which satisfy the Sil'nikov inequality given by

$$
\omega_{k} \neq 0, \quad \sigma_{k} \delta_{k}<0, \quad\left|\delta_{k}\right|>\left|\sigma_{k}\right|>0, \quad k=1,2,
$$

with the further constraint

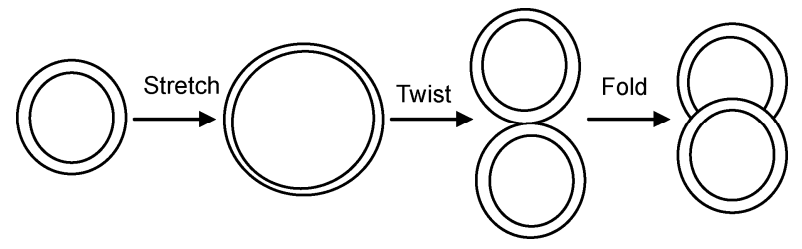

Figure 1 The stretch-twist-fold sequence.

$$
\sigma_{1} \sigma_{1}>0 \text { or } \delta_{1} \delta_{1}>0
$$

Suppose a heteroclinic orbit exists, which joins $x^{1}$ and $x^{2}$, it gives

(i) The Sil'nikov map, has a countable number of Smale horseshoes in its discrete dynamics, which is defined in a neighborhood of the heteroclinic orbit.

(ii) For any sufficiently small $C^{1}$-perturbation $h$ of $f$, the perturbed system

$$
\frac{\mathrm{d} x}{\mathrm{~d} t}=h(x), \quad x \in R^{3}
$$

defined near the heteroclinic orbit, has at least a finite number of Smale horseshoes in the discrete dynamics of Sil'nikov map.

(iii) The perturbed system (4) and original system (1) both have horseshoes type of chaos.

For convenience, a heteroclinic orbit satisfying (2) and (3) is referred to as Sil'nikov type. The heteroclinic Sil'nikov criterion implies that if system (1) has one heteroclinic orbit of Sil'nikov type, which connects two distinct saddle foci of the system, then it has Smale horseshoes chaos.

\section{The stretch-twist-fold (STF) flow model}

STF is steady incompressible flow in a unit sphere, which is a prototype of the stretch-twist-fold mechanism of the magnetic field generation [7-9] and given as

$$
\begin{aligned}
& \dot{x}(t)=\alpha z-8 x y, \\
& \dot{y}(t)=11 x^{2}+3 y^{2}+z^{2}+\beta x z-3, \\
& \dot{z}(t)=-\alpha x+2 y z-\beta x y,
\end{aligned}
$$

where $(x, y, z) \in R^{3}$ and $\alpha, \beta \in R$ are positive real parameters which show the relationship of the ratios of intensities of stretch, twist and fold ingredients of flow [6-8]. It can be observed that STF flow $u=\left(\alpha z-8 x y, 11 x^{2}+3 y^{2}\right.$ $\left.+z^{2}+\beta x z-3,-\alpha x+2 y z-\beta x y\right) \quad$ satisfies $\quad \nabla \cdot u=0 ;\left.u \cdot n\right|_{S}$ $=0$, where $S$ is the surface of the unit sphere [6-8]. When $\alpha=0.2, \beta=2$, its behavior is chaotic as shown in Figure 2, meanwhile the chaotic time series of trajectories $x(t), y(t)$, $z(t)$ appear in Figure 3.

\section{Parameter analysis of STF flow}

Parameter analysis of STF-flow is considered only for equilibrium points because at equilibrium points the behavior of the system remains constant with the passage of time. Now we discuss the possible choices of the parameters values.

Case I If $\alpha=0, \beta=0$, system (5) has two equilibria: $p_{1}=(0,1,0)$ and $p_{2}=(0,-1,0)$. 

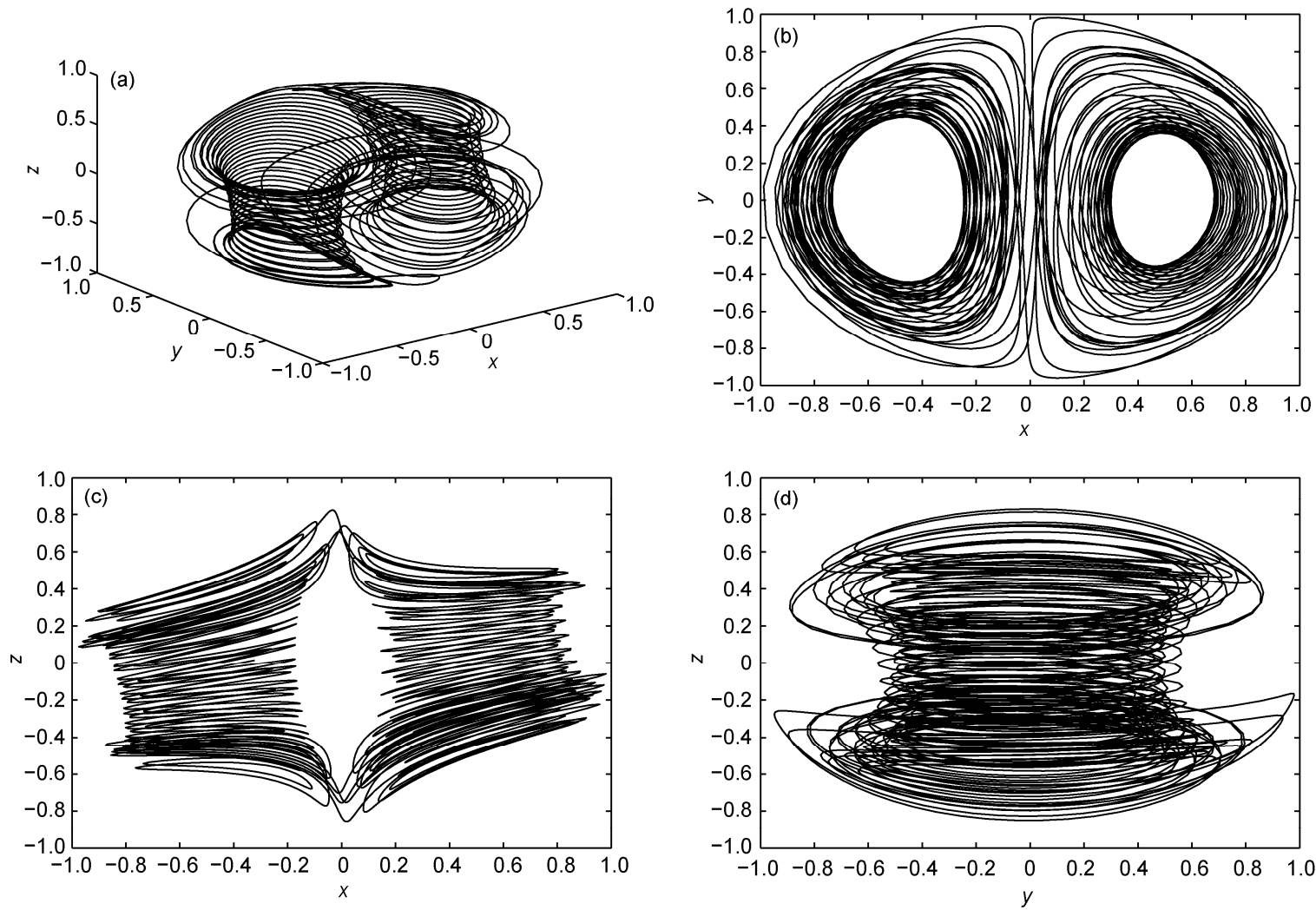

Figure 2 Phase portrait of chaotic system (5) with $\alpha=0.2, \beta=2$. (a) In $x-y-z$ space; (b) in $x-y$ plane; (c) in $x-z$ plane; (d) in $y-z$ plane.
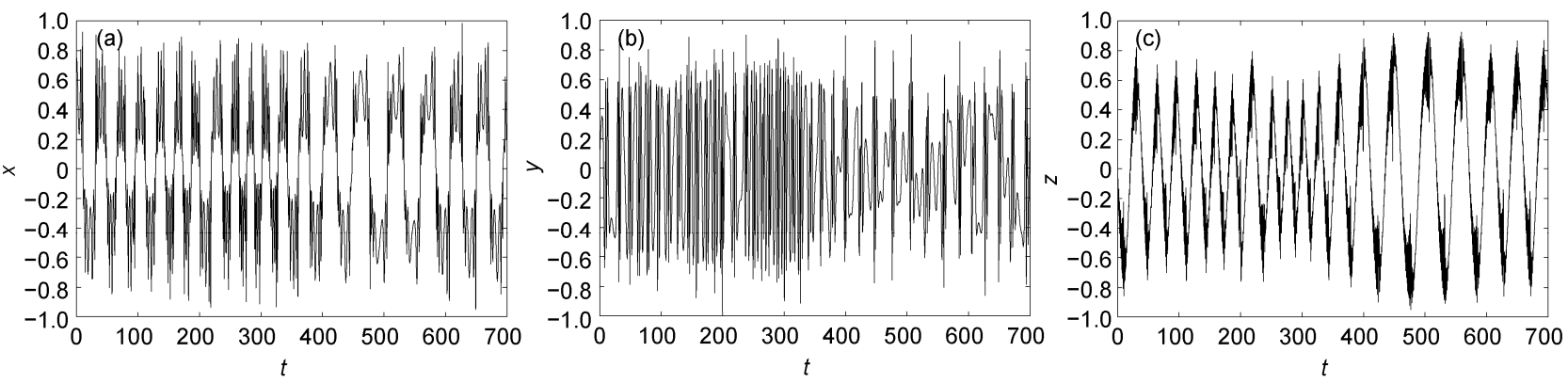

Figure 3 Chaotic time histories of trajectory $x(t)(\mathrm{a}), y(t)(\mathrm{b})$ and $z(t)(\mathrm{c})$.

The Jacobian matrix of system (5) evaluated at $p_{1}$ gives characteristic equation as $\lambda^{3}-52 \lambda+96=0$, that has roots: $\lambda_{1}=6, \lambda_{2}=2, \lambda_{3}=-8$, so $p_{1}$ is a saddle.

The characteristic equation evaluated at $p_{2}$ is $\lambda^{3}-52 \lambda$ $-96=0$, which has following roots: $\lambda_{1}=-6, \lambda_{2,3}=3 \pm \sqrt[2]{4} i$, so $p_{2}$ is a saddle focus.

Case II If $\alpha=0, \beta>0$, system (5) has two equilibria: $p_{3}=(0,1,0)$ and $p_{4}=(0,-1,0)$, which is the same as case I, so $p_{3}$ is saddle and $p_{4}$ is saddle focus.

Case III If $\alpha>0, \beta=0$, system (5) has six equilibria for $16-\alpha^{2}>0$ :

$$
p_{5}=(0,1,0), p_{6}=(0,-1,0) \text {, }
$$

$$
\begin{aligned}
& p_{7}=\left(\frac{1}{4} \sqrt{\frac{16-\alpha^{2}}{5}},-\frac{\alpha}{4},-\frac{1}{2} \sqrt{\frac{16-\alpha^{2}}{5}}\right), \\
& p_{8}=\left(-\frac{1}{4} \sqrt{\frac{16-\alpha^{2}}{5}},-\frac{\alpha}{4}, \frac{1}{2} \sqrt{\frac{16-\alpha^{2}}{5}}\right), \\
& p_{9}=\left(\frac{1}{4} \sqrt{\frac{16-\alpha^{2}}{5}}, \frac{\alpha}{4}, \frac{1}{2} \sqrt{\frac{16-\alpha^{2}}{5}}\right), \\
& p_{10}=\left(-\frac{1}{4} \sqrt{\frac{16-\alpha^{2}}{5}}, \frac{\alpha}{4},-\frac{1}{2} \sqrt{\frac{16-\alpha^{2}}{5}}\right) .
\end{aligned}
$$

Characteristic equation evaluated at $p_{5}$ is $\lambda^{3}-(52$ 
$\left.-\alpha^{2}\right) \lambda+\left(96-6 \alpha^{2}\right)=0$ that has roots: $\lambda_{1}=6, \lambda_{2,3}$ $=-3 \pm \sqrt{25-\alpha^{2}}$. If $25-\alpha^{2} \geqslant 0$, then $p_{5}$ has saddle and if $25-\alpha^{2}<0$, then $p_{5}$ has saddle focus.

Characteristic equation evaluated at $p_{6}$ is $\lambda^{3}-(52$ $\left.-\alpha^{2}\right) \lambda+\left(-96+6 \alpha^{2}\right)=0$ that has roots: $\lambda_{1}=-6, \lambda_{2,3}=$ $3 \pm \sqrt{25-\alpha^{2}}$. If $25-\alpha^{2} \geqslant 0$, then $p_{6}$ is a saddle and if $25-\alpha^{2}<0$, then $p_{6}$ is a saddle focus.

Characteristic equation evaluated at $p_{7}$ is $\lambda^{3}-\left(\frac{17}{4} \alpha^{2}-32\right) \lambda-\left(3 \alpha^{3}-48 \alpha\right)=0$ that contains roots: $\lambda_{1}=-\frac{3}{2} \alpha, \lambda_{2,3}=\frac{3}{4} \pm \sqrt{41 \alpha^{2}-512}$. If $41 \alpha^{2}-512 \geqslant 0$, then $p_{7}$ is a saddle and if $41 \alpha^{2}-512<0$, then $p_{7}$ is a saddle focus.

Characteristic equation evaluated at $p_{8}$ is the same as for equilibrium point $p_{7}$, so if $41 \alpha^{2}-512 \geqslant 0$, then $p_{8}$ is a saddle and if $41 \alpha^{2}-512<0$, then $p_{8}$ is a saddle focus.

Characteristic equation evaluated at $p_{9}$ is $\lambda^{3}-\left(\frac{17}{4} \alpha^{2}-32\right) \lambda+\left(3 \alpha^{3}-48 \alpha\right)=0$ that has roots: $\lambda_{1}=\frac{3}{2} \alpha, \lambda_{2,3}=-\frac{3}{4} \pm \sqrt{41 \alpha^{2}-512}$. If $41 \alpha^{2}-512 \geqslant 0$, then $p_{9}$ is a saddle and if $41 \alpha^{2}-512<0$, then $p_{9}$ is a saddle focus.

Characteristic equation evaluated at $p_{10}$ is the same as for equilibrium point $p_{9}$, so if $41 \alpha^{2}-512 \geqslant 0$, then $p_{10}$ is a saddle and if $41 \alpha^{2}-512<0$, then $p_{10}$ is a saddle focus.

Case IV If $\alpha>0, \beta>0$, system (5) has six equilibria for $J / F>0, K / G>0$ :

$$
\begin{aligned}
& p_{11}=(0,1,0), p_{12}=(0,-1,0), \\
& p_{13}=\left(\frac{1}{8} \sqrt{\frac{J}{F}},-\frac{H \alpha}{32},-\frac{H}{32} \sqrt{\frac{J}{F}}\right), \\
& p_{14}=\left(-\frac{1}{8} \sqrt{\frac{J}{F}},-\frac{H \alpha}{32}, \frac{H}{32} \sqrt{\frac{J}{F}}\right), \\
& p_{15}=\left(\frac{1}{8} \sqrt{\frac{K}{G}}, \frac{I \alpha}{32}, \frac{I}{32} \sqrt{\frac{K}{G}}\right),
\end{aligned}
$$

$$
p_{16}=\left(-\frac{1}{8} \sqrt{\frac{K}{G}}, \frac{I \alpha}{32},-\frac{I}{32} \sqrt{\frac{K}{G}}\right) .
$$

where

$$
\begin{aligned}
& F=-\beta^{2}-40+\beta \sqrt{64+\beta^{2}}, \\
& G=\beta^{2}+40+\beta \sqrt{64+\beta^{2}}, \\
& H=-\beta+\sqrt{64+\beta^{2}}, \\
& I=\beta+\beta \sqrt{64+\beta^{2}}, \\
& J=32 \alpha^{2}+\alpha^{2} \beta^{2}-\alpha^{2} \beta \sqrt{64+\beta^{2}}-512, \\
& K=-32 \alpha^{2}-\alpha^{2} \beta^{2}-\alpha^{2} \beta \sqrt{64+\beta^{2}}+512 .
\end{aligned}
$$

Characteristic equation evaluated at $p_{11}$ is $\lambda^{3}+$ $\left(-52-\alpha^{2}+\alpha \beta\right) \lambda+\left(96-6 \alpha^{2}-6 \alpha \beta\right)=0$ that has roots: $\lambda_{1}=6, \lambda_{2,3}=-3 \pm \sqrt{25-\alpha^{2}-\alpha \beta}$. Therefore if $25-\alpha^{2}$ $-\alpha \beta \geqslant 0$, then $p_{11}$ is a saddle and if $25-\alpha^{2}-\alpha \beta<0$, then $p_{11}$ is a saddle focus.

Characteristic equation evaluated at $p_{12}$ is $\lambda^{3}+\left(-52-\alpha^{2}+\alpha \beta\right) \lambda+\left(-96+6 \alpha^{2}+6 \alpha \beta\right)=0$ that has roots: $\lambda_{1}=6, \lambda_{2,3}=-3 \pm \sqrt{25-\alpha^{2}-\alpha \beta}$. Therefore if 25 $-\alpha^{2}-\alpha \beta \geqslant 0$, then $p_{12}$ is a saddle and if $25-\alpha^{2}-\alpha \beta<0$, then $p_{12}$ is a saddle focus.

The Jacobian matrix of system (5), evaluated at $p_{13}$ is

$$
J_{p_{13}}=\left(\begin{array}{ccc}
\frac{H \alpha}{4}-\lambda & -\sqrt{\frac{J}{F}} & \alpha \\
\frac{11}{4} \sqrt{\frac{J}{F}}-\frac{\beta H}{32} \sqrt{\frac{J}{F}} & -\frac{3}{16} H \alpha-\lambda & -\frac{H}{16} \sqrt{\frac{J}{F}}+\frac{\beta}{8} \sqrt{\frac{J}{F}} \\
-\alpha+\frac{\alpha \beta H}{32} & -\frac{H}{16} \sqrt{\frac{J}{F}}-\frac{\beta}{8} \sqrt{\frac{J}{F}} & -\frac{H \alpha}{16}-\lambda
\end{array}\right)
$$

Characteristic equation of $J_{p_{13}}$ is

$$
\lambda^{3}+p \lambda+q=0
$$

where

$$
\begin{aligned}
p= & -\frac{1}{-40-\beta^{2}+\sqrt{64+\beta^{2}}}\left(-\frac{\beta^{3}}{16}+\frac{\beta^{2} \sqrt{64+\beta^{2}}}{16}-\frac{21 \beta}{4}-\frac{80}{\beta}+\frac{13 \sqrt{64+\beta^{2}}}{4}\right) \alpha^{2}+\left(\frac{9 \beta^{2}}{128}-\frac{9 \beta \sqrt{64+\beta^{2}}}{128}+\frac{9}{4}\right) \alpha^{2} \\
& +\frac{1}{-40-\beta^{2}+\sqrt{64+\beta^{2}}}\left(20 \beta+\frac{1280}{\beta}-12 \sqrt{64+\beta^{2}}\right), \\
q= & -\frac{1}{-40-\beta^{2}+\sqrt{64+\beta^{2}}}\left(\frac{3 \beta^{4}}{128}-\frac{3 \beta^{3} \sqrt{64+\beta^{2}}}{128}+\frac{75 \beta^{2}}{32}-\frac{51 \sqrt{64+\beta^{2}}}{32}-\frac{15 \sqrt{64+\beta^{2}}}{\beta}+54\right) \alpha^{3} \\
& -\frac{1}{-40-\beta^{2}+\sqrt{64+\beta^{2}}}\left(-6 \beta^{2}+6 \beta \sqrt{64+\beta^{2}}+\frac{240 \sqrt{64+\beta^{2}}}{\beta}-384\right) \alpha .
\end{aligned}
$$


Observe that the term $\lambda^{2}$ is missing in the characteristic polynomial (7), so we use the Cardano's formula to solve this cubic equation. According to Cardano's formula, we have

$$
\Delta=\left(\frac{q}{2}\right)^{2}+\left(\frac{p}{3}\right)^{3}
$$

When $\Delta>0$, eq. (7) has a unique real root, $\lambda_{1}=\delta_{1}$ and one conjugate pair of complex roots, $\lambda_{2}=\sigma_{1}=i \omega_{1}$ and $\lambda_{3}=\sigma_{1}=i \omega_{1}$, where $i=\sqrt{-1}, \delta_{1}=\mu_{1}+v_{1}, \sigma_{1}=-\frac{\mu_{1}+v_{1}}{2}$, $\omega_{1}=\frac{\sqrt{3}}{2}\left(\mu_{1}-v_{1}\right)$, with $\mu_{1}=\sqrt[3]{-\frac{q}{2}+\sqrt{\Delta}}, v_{1}=\sqrt[3]{\frac{q}{2}-\sqrt{\Delta}}$.
Obviously, $\mu_{1}+v_{1} \neq 0$ and $\mu_{1}-v_{1} \neq 0$, if $\Delta>0$.

If $\Delta>0$, then $p_{13}$ is a saddle focus and if $\Delta<0$, then $p_{13}$ is a saddle.

Observe that, the Jacobian matrix of system (5) evaluated at equilibrium point $p_{14}$ gives the same characteristic equation as in eq. (7). Therefore if $\Delta>0$, then $p_{14}$ is a saddle focus and if $\Delta<0$, then $p_{14}$ is a saddle node.

Characteristic equation of $J_{p_{15}}$ is

$$
\lambda^{3}+p_{1} \lambda+q_{1}=0
$$

$$
\begin{aligned}
p_{1}= & -\frac{1}{40+\beta^{2}+\sqrt{64+\beta^{2}}}\left(\frac{\beta^{3}}{16}+\frac{\beta^{2} \sqrt{64+\beta^{2}}}{16}+\frac{21 \beta}{4}+\frac{80}{\beta}+\frac{13 \sqrt{64+\beta^{2}}}{4}\right) \alpha^{2}-\left(\frac{9 \beta^{2}}{128}+\frac{9 \beta \sqrt{64+\beta^{2}}}{128}+\frac{9}{4}\right) \alpha^{2} \\
& +\frac{1}{40+\beta^{2}+\sqrt{64+\beta^{2}}}\left(20 \beta+\frac{1280}{\beta}+12 \sqrt{64+\beta^{2}}\right), \\
q_{1}= & \frac{1}{40+\beta^{2}+\sqrt{64+\beta^{2}}}\left(\frac{3 \beta^{4}}{128}+\frac{3 \beta^{3} \sqrt{64+\beta^{2}}}{128}+\frac{75 \beta^{2}}{32}+\frac{51 \sqrt{64+\beta^{2}}}{32}+\frac{15 \sqrt{64+\beta^{2}}}{\beta}+54\right) \alpha^{3} \\
& -\frac{1}{40+\beta^{2}+\sqrt{64+\beta^{2}}}\left(6 \beta^{2}+6 \beta \sqrt{64+\beta^{2}}+\frac{240 \sqrt{64+\beta^{2}}}{\beta}+384\right) \alpha .
\end{aligned}
$$

Observe that the term $\lambda^{2}$ is missing in the characteristic polynomial (9), so we use Cardano's formula to solve this cubic equation. According to Cardano's formula, we have

$$
\Delta_{1}=\left(\frac{q_{1}}{2}\right)^{2}+\left(\frac{p_{1}}{3}\right)^{3}
$$

When $\Delta_{1}>0$, eq. (9) has a unique real root $\lambda_{1}=\delta_{2}$, and one conjugate pair of complex roots $\lambda_{2}=\sigma_{2}=i \omega_{2}$ and $\lambda_{3}=$ $\sigma_{2}=i \omega_{2}$, where $i=\sqrt{-1}, \delta_{2}=\mu_{2}+v_{2}, \sigma_{2}=-\frac{\mu_{2}+v_{2}}{2}, \omega_{2}=$ $\frac{\sqrt{3}}{2}\left(\mu_{2}-v_{2}\right)$, with $\mu_{2}=\sqrt[3]{-\frac{q_{1}}{2}+\sqrt{\Delta_{1}}}, v_{2}=\sqrt[3]{-\frac{q_{1}}{2}-\sqrt{\Delta_{1}}}$. Obviously, $\mu_{2}+v_{2} \neq 0$ and $\mu_{2}-v_{2} \neq 0$, if $\Delta_{1}>0$.

If $\Delta_{1}>0$, then $p_{15}$ is a saddle focus and if $\Delta_{1}<0$, then $p_{15}$ is a saddle.

Observe that the Jacobian matrix of system (5) evaluated at the equilibrium point $p_{16}$ gives the same characteristic equation as eq. (9). Therefore if $\Delta_{1}>0$, then $p_{16}$ is a saddle focus and if $\Delta_{1}<0$, then $p_{16}$ is a saddle.

\section{Series expansion of Heteroclinic orbit and its convergence}

From Figure 2, we see that in chaotic STF flow (5), the trajectories are swirling between the two equilibria. Therefore we can assume that a Heteroclinic orbit which joins two equilibria may exist there. To prove the existence of heteroclinic orbit of the system (5), which links two equilibria $p_{13}$ and $p_{14}$, we apply the undetermined coefficient method and present the exact algebraic expressions of Heteroclinic orbit. Without loss of generality, one may stipulate a definite direction as follows: from $p_{13}$ to $p_{14}$ corresponds to forward asymptotic time $t \rightarrow+\infty$, while from $p_{14}$ to $p_{13}$ corresponds to reverse asymptotic time $t \rightarrow-\infty$.

In case of $t>0$, let

$$
\begin{aligned}
& x(t)=x_{13}+\sum_{k=1}^{\infty} a_{k} e^{k \eta t}, \\
& y(t)=y_{13}+\sum_{k=1}^{\infty} b_{k} e^{k \eta t}, \\
& z(t)=z_{13}+\sum_{k=1}^{\infty} c_{k} e^{k \eta t},
\end{aligned}
$$

where $\eta<0$ is undetermined constant, $a_{k}, b_{k}, c_{k}(k \geqslant 0)$ are also undetermined coefficients. Substituting (11) into (5) gives

$$
\begin{aligned}
& a_{1} \eta e^{\eta t}+2 a_{2} \eta e^{2 \eta t}+3 a_{3} \eta e^{3 \eta t}+\ldots \\
= & \left(-8 b_{1} x_{13}-8 a_{1} y_{13}+\alpha c_{1}\right) e^{\eta t} \\
& +\left(-8 b_{2} x_{13}-8 a_{2} y_{13}-8 a_{1} b_{1}+\alpha c_{2}\right) e^{2 \eta t} \\
& +\left(-8 b_{3} x_{13}-8 a_{3} y_{13}-8 a_{1} b_{1}-8 a_{2} b_{2}+\alpha c_{3}\right) e^{3 \eta t}+\ldots
\end{aligned}
$$




$$
\begin{aligned}
& b_{1} \eta e^{\eta t}+2 b_{2} \eta e^{2 \eta t}+3 b_{3} \eta e^{3 \eta t}+\ldots \\
&=\left(22 a_{1} x_{13}+\beta c_{1} x_{13}-6 b_{1} y_{13}+2 c_{1} z_{13}+\beta a_{1} z_{13}\right) e^{\eta t} \\
&+\left(22 a_{2} x_{13}+\beta c_{2} x_{13}+6 b_{2} y_{13}+2 c_{2} z_{13}+\beta a_{2} z_{13}\right. \\
&\left.+11 a_{1}^{2}+3 b_{1}^{2}+c_{1}^{2}+\beta a_{1} c_{2}\right) e^{2 \eta t} \\
&+\left(22 a_{3} x_{13}+\beta c_{3} x_{13}+6 b_{3} y_{13}+2 c_{3} z_{13}+\beta a_{3} z_{13}\right. \\
&\left.+22 a_{2}^{2}+6 b_{2}^{2}+2 c_{2}^{2}+\beta a_{1} c_{2}+\beta a_{2} c_{1}\right) e^{3 \eta t}+\ldots \\
& c_{1} \eta e^{\eta t}+2 c_{2} \eta e^{2 \eta t}+3 c_{3} \eta e^{3 \eta t}+\ldots \\
&=\left(-\beta b_{1} x_{13}+2 c_{1} y_{13}-\beta a_{1} y_{13}+2 b_{1} z_{13}-\alpha a_{1}\right) e^{\eta t} \\
&+\left(-\beta b_{2} x_{13}+2 c_{2} y_{13}-\beta a_{2} y_{13}+2 b_{2} z_{13}+2 b_{1} c_{1}\right. \\
&\left.-\alpha a_{2}-\beta a_{1} b_{1}\right) e^{2 \eta t} \\
&+\left(-\beta b_{3} x_{13}+2 c_{3} y_{13}-\beta a_{3} y_{13}+2 b_{3} z_{13}+2 b_{1} c_{2}\right. \\
&\left.+2 b_{2} c_{1}-\alpha a_{3}-\beta a_{1} b_{2}-\beta a_{2} b_{1}\right) e^{3 \eta t}+\ldots
\end{aligned}
$$

Now, comparing the coefficients of like powers of $e^{k \eta t}$, for $k=1$, in system (12)-(14), we have

$$
\left[\begin{array}{ccc}
\eta+8 y_{13} & 8 x_{13} & -\alpha \\
-22 x_{13}-\beta z_{13} & \eta-6 y_{13} & -2 z_{13}-\beta x_{13} \\
\alpha+\beta y_{13} & -2 z_{13}+\beta x_{13} & \eta-2 y_{13}
\end{array}\right]\left[\begin{array}{l}
a_{1} \\
b_{1} \\
c_{1}
\end{array}\right]=\left[\begin{array}{l}
0 \\
0 \\
0
\end{array}\right] .
$$

It can be written as

$$
\left[\eta I-J_{p_{13}}\right]\left[\begin{array}{l}
a_{1} \\
b_{1} \\
c_{1}
\end{array}\right]=\left[\begin{array}{l}
0 \\
0 \\
0
\end{array}\right]
$$

where $J_{p_{13}}$ is the Jacobian of system (5) evaluated at the equilibrium point $p_{13}$.

Now for $k \geqslant 2$, we have

$$
\left[k \eta I-J_{p_{13}}\right]\left[\begin{array}{l}
a_{k} \\
b_{k} \\
c_{k}
\end{array}\right]=\left[\begin{array}{c}
\sum_{i=1}^{k-1}\left(-8 a_{i} b_{k-i}\right) \\
\sum_{i=1}^{k-1}\left(11 a_{i} a_{k-i}+3 b_{i} b_{k-i}+c_{i} c_{k-i}+\beta a_{i} c_{k-i}\right) \\
\sum_{i=1}^{k-1}\left(2 b_{i} c_{k-i}-\beta a_{i} b_{k-i}\right)
\end{array}\right] .
$$

Assume that $\left(a_{1}, b_{1}, c_{1}\right) \neq(0,0,0)$, otherwise, we can gain $\left(a_{k}, b_{k}, c_{k}\right)=(0,0,0)$ for all $k>1$. In this case, it might be surprising to observe that in eq. (15), $J_{p_{13}}$ is the Jacobian of the linearized system at the equilibrium point $p_{13}$. Since $J_{p_{13}}$ has the unique negative $\eta$, so that $\operatorname{det}\left(\eta I-J_{p_{13}}\right)=0 . \quad$ If $\quad \operatorname{det}\left(\eta I-J_{p_{13}}\right)=0, \quad$ then $\operatorname{ker}\left(\eta I-J_{p_{13}}\right) \neq 0$, it implies that there exist non zero values of $a_{1}, b_{1}$ and $c_{1}$. Therefore if $\operatorname{det}\left(\eta I-J_{p_{13}}\right)=0$, then $\left(a_{1}, b_{1}, c_{1}\right) \neq(0,0,0)$. Note that $\operatorname{det}\left(k \eta I-J_{p_{13}}\right) \neq 0$ for $k>1$. From eqs. (15) and (16), $a_{k}(k>1), \quad b_{k}(k>1)$ and $c_{k}(k>1)$ can be determined by $\eta, \alpha, \beta, a_{1}, b_{1}$ and $c_{1}$.

For $t>0$, the first part of the Heteroclinic orbit is established. For remaining part, one can assume that for $t<0$

$$
\begin{aligned}
& x(t)=x_{14}-\sum_{k=1}^{\infty} a_{k} e^{-k \zeta t}, \\
& y(t)=y_{14}-\sum_{k=1}^{\infty} b_{k} e^{-k \zeta t}, \\
& z(t)=z_{14}-\sum_{k=1}^{\infty} c_{k} e^{-k \zeta t},
\end{aligned}
$$

where $\zeta<0$ is undetermined constant, $a_{k}, b_{k}, c_{k}(k \geqslant 1)$ are also undetermined coefficients.

As in case of $t>0$, one can obtain $\eta=\zeta$. Also from the continuity of the solution at $t=0$, from eq. (11) and eq. (17), we have

$$
\begin{aligned}
& x_{13}+\sum_{k=1}^{\infty} a_{k}=x_{14}-\sum_{k=1}^{\infty} a_{k}, \\
& y_{13}+\sum_{k=1}^{\infty} b_{k}=y_{14}-\sum_{k=1}^{\infty} b_{k}, \\
& z_{13}+\sum_{k=1}^{\infty} c_{k}=z_{14}-\sum_{k=1}^{\infty} c_{k} .
\end{aligned}
$$

It determines the values of $a_{1}, b_{1}$ and $c_{1}$. Consequently, $a_{k}(k>1), \quad b_{k}(k>1)$ and $c_{k}(k>1)$ have been completely determined by $a_{1}, b_{1}, c_{1}, \eta=\zeta, \alpha$ and $\beta$. Thus, if $\alpha$ and $\beta$ satisfy some conditions (for example, $\alpha=0.2$ and $\beta=2$ ), STF flow has following Heteroclinic orbit:

$$
x(t)= \begin{cases}x_{13}+\sum_{k=1}^{\infty} a_{k} e^{k \eta t}, & t>0, \\ 0, & t=0, \\ x_{14}-\sum_{k=1}^{\infty} a_{k} e^{-k \eta t}, & t<0,\end{cases}
$$$$
y(t)= \begin{cases}y_{13}+\sum_{k=1}^{\infty} b_{k} e^{k \eta t}, & t>0, \\ 0, & t=0, \\ y_{14}-\sum_{k=1}^{\infty} b_{k} e^{-k \eta t}, & t<0,\end{cases}
$$

$$
z(t)= \begin{cases}z_{13}+\sum_{k=1}^{\infty} c_{k} e^{k \eta t}, & t>0, \\ 0, & t=0, \\ z_{14}-\sum_{k=1}^{\infty} c_{k} e^{-k \eta t}, & t>0,\end{cases}
$$


which joins the equilibria $p_{13}$ and $p_{14}$.

Now the convergence of the Heteroclinic orbit series expansion (11) is taken into an account. Here, we consider the case of STF flow with typical parameter set that generates chaotic behavior. STF flow is chaotic with typical parameters $\alpha=0.2, \beta=2$. In this case $x_{13}=0.538874495, y_{13}=$ $-0.03903882, z_{13}=-0.849913082$ and $\eta=\zeta=-0.236043$ is determined by eq. (15). Then $a_{k}(k \geqslant 1), b_{k}(k \geqslant 1)$ and $c_{k}(k \geqslant 1)$ can be observed by eqs. (15) and (16). Notice that $a_{k}(k \geqslant 1), b_{k}(k \geqslant 1)$ and $c_{k}(k \geqslant 1)$ are bounded. There exists a $M>0$, such that $\left|a_{k}\right| \leqslant M,\left|b_{k}\right| \leqslant M$ and $\left|c_{k}\right| \leqslant M, k=1,2, \ldots$ then

$$
\begin{aligned}
& \sum_{k=1}^{\infty}\left|a_{k} e^{k \eta t}\right| \leqslant M \sum_{k=0}^{\infty} a_{k} e^{k \eta t}, t>0, \\
& \sum_{k=1}^{\infty}\left|b_{k} e^{k \eta t}\right| \leqslant M \sum_{k=0}^{\infty} b_{k} e^{k \eta t}, t>0, \\
& \sum_{k=1}^{\infty}\left|c_{k} e^{k \eta t}\right| \leqslant M \sum_{k=0}^{\infty} b_{k} e^{k \eta t}, t>0 .
\end{aligned}
$$

Obviously $\quad x_{13}+\sum_{k=0}^{\infty} a_{k} e^{k \eta t}, \quad y_{13}+\sum_{k=0}^{\infty} b_{k} e^{k \eta t} \quad$ and $z_{13}+$ $\sum_{k=0}^{\infty} c_{k} e^{k \eta t}$ are convergent on $(0,+\infty)$. In the same way, we can prove that $x_{14}-\sum_{k=0}^{\infty} a_{k} e^{-k \zeta t}, \quad y_{14}-\sum_{k=0}^{\infty} b_{k} e^{-k \zeta t} \quad$ and $z_{14}-\sum_{k=0}^{\infty} c_{k} e^{-k \zeta t} \quad$ are convergent on $(-\infty, 0)$.

System (5) has a Heteroclinic orbit that links $p_{13}$ and $p_{14}$ with the suitable parameters $\alpha=0.2, \beta=2$, which is in the form of (19)-(21), which is shown in Figure 4. The proof is the same for other values of parameters, if Heteroclinic orbit exists.

\section{Existence of conjugate STF trajectory}

From Sil'nikov theorem, we can infer a result that if STF

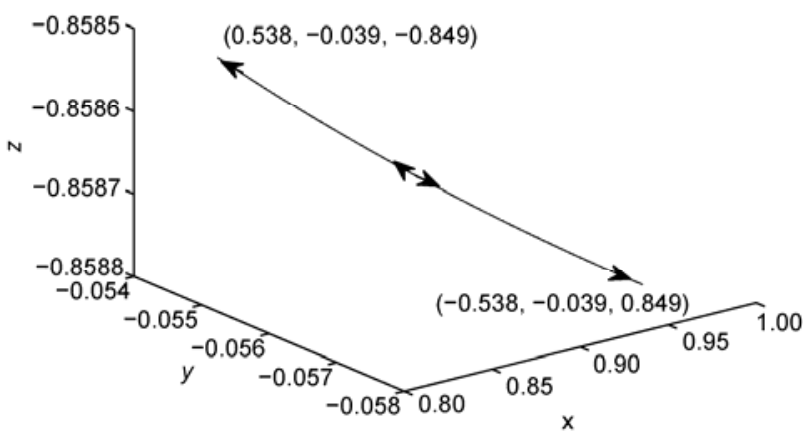

Figure 4 Heteroclinic orbit of STF flow for $\alpha=0.2, \beta=2$. system (5) has two saddle foci that fulfill the criteria (2) and (3) and a Heteroclinic orbit that links two equilibria, then STF system (5) has horseshoes chaos. First of all we discuss the presence of chaotic trajectory of system (5) with the suitable parameters. When $\alpha=0.2$ and $\beta=2$ at equilibria $p_{13}\left(p_{14}\right)$, we have the following three eigen values: $\lambda_{1}=$ $-0.234233, \lambda_{2}=0.117116-6.4937 i, \lambda_{3}=0.117116+6.4937 \mathrm{i}$, which satisfy $|-0.234233|>0.117116$. Therefore STF flow (5) has two saddle foci $p_{13}$ and $p_{14}$. In the previous section, we proved the presence of Heteroclinic orbit that links $p_{13}$ and $p_{14}$; thus STF flow (5) has Smale horseshoes type chaos.

Now we enhance this idea for other values of parameters $\alpha, \beta$, so to prove the Sil'nikov inequalities, we need to prove $\Delta=\left(\frac{q}{2}\right)^{2}+\left(\frac{p}{3}\right)^{3}>0$, we calculated $\Delta=\left(\frac{q}{2}\right)^{2}+\left(\frac{p}{3}\right)^{3}$, where $p$ and $q$ are given in Section 3, from this we deduced the result that if

$$
\begin{aligned}
\alpha^{2}< & {\left[75 \beta^{5}-50 \beta^{4}+6768 \beta^{3}-4512 \beta^{2}+125952 \beta\right.} \\
+ & \left(75 \beta^{4}-50 \beta^{3}+4368 \beta^{2}+2912 \beta\right) \sqrt{\beta^{2}+64} \\
& -83968] /\left(150 \beta^{3}-100 \beta^{2}+10086 \beta-6724\right),
\end{aligned}
$$

then $\Delta=\left(\frac{q}{2}\right)^{2}+\left(\frac{p}{3}\right)^{3}$ is positive. For other parameters if they satisfy $\alpha>0, \beta>0$ and $\Delta=\left(\frac{q}{2}\right)^{2}+\left(\frac{p}{3}\right)^{3}>0$, system (5) has two saddle foci that fulfill the Sil'nikov criteria (2) and (3). Note that, this condition obviously satisfies for the typical parameter set.

\section{Conclusions}

In our analysis, one Heteroclinic orbit of system (5) with typical parameter sets is indentified using undetermined coefficient method. With typical parameter values of $\alpha=0.2$, $\beta=2$, it is shown that the STF flow has Smale horseshoes chaos. Parameter analysis is given for different choices of parameters. It is important to note, numerically it is difficult to identify the Heteroclinic orbit of chaotic system. Use of undetermined coefficient method provides a powerful tool for doing so. Based on Sil'nikov theorem, we proved that typical values of parameters which satisfy some conditions chaos can exist in STF flow.

Chaotic behavior of the STF flow is investigated. Not so much research has been done on this system, so this work can be extended to explore further complex dynamical behavior of STF flow. Results obtained from the STF flow are useful to explore the magnetic field structure in plasma and astronomy. 
This work was supported by the National Natural Science Foundation of China (10772026, 11072030), the Ph.D. Programs Foundation of Ministry of Education of China (20080070011), the Scientific Research Foundation of Ministry of Education of China for Returned Scholars (20080732040) and the Program of Beijing Municipal Key Discipline Construction.

1 Wang J, Chen Z, Yuan Z. Existence of a new three-dimensional chaotic attractor. Chaos Solitons Fractals, 2009, 42: 3053-3057

2 Liu N, Guan Z H. Chaotification for a class of non linear system. Chin Phys B, 2009, 18: 1769-1773

3 Yang N, Long Z C, Zhao X H. Method for removing interface in chaotic signal based on the Lyapunov exponent. Chin Sci Bull, 2012, 57: 455-459

4 Chen G, Dong X. From Chaos to Order: Methodologies, Perspectives and Applications. Singapore: World Scientific Pub. Co, 1998

5 Wang X. Relation of chaos activity characteristics of the cardiac system with the evolution of species. Chin Sci Bull, 2002, 47: 20422048

6 Vaĭnshteĭn S I, Zel'dovich Ya B. Origin of magnetic fields in astrophysics (turbulent "dynamo" mechanisms). Sov Phys Usp, 1972, 15: 159-172

7 Moffatt H K, Proctor M R E. Topological constraints associated with fast dynamo action. J Fluid Mech, 1985, 154: 493-507

8 Bajer K, Moffatt H K. On a class of steady confined stokes flows with chaotic streamlines. J Fluid Mech, 1990, 212: 337-363

9 Bajer K. Flow kinematics and magnetic equilibria. Dissertation for Doctoral Degree. Cambridge: University of Cambridge, 1989

10 Bajer K, Moffatt H K, Nex F H. Steady confined stokes flows with chaotic streamlines. In: Moffatt H K, Tsinober A, eds. Topological Fluid Mechanics. Proceedings of the IUTAM Symposium. Cambridge: Cambridge University Press, 1990. 459-466

11 Streetman B, Peck M A. New synchronous orbits using the geomagnetic Lorentz force. J Guid Contr Dyn, 2007, 30: 1677-1690
12 Victoria L C, John E P. Technique for escape from geosynchronous transfer orbit using a solar sail. J Guid Contr Dyn, 2003, 26: 628-634

13 Phipps C, Luke J, Lippert T, et al. Micropropulsion using a laser ablation jet. J Propul Power, 2004, 20: 1000-1011

14 Li R, Xu B, Zhang H. Dynamic feature analysis of space electrodynamics tether system. J Vibrat Shock, 2008, 27: 36-40

15 Yue B Z, Wang Z. Nonlinear phenomena of three-dimensional liquid sloshing in microgravity environment. Chin Sci Bull, 2006, 51: 2425-2431

16 Lorenz E N. Deterministic non periodic flow. J Atoms Sci, 1963, 20: $130-141$

17 Lü J H, Chen G R. A new chaotic attractor coined. Int J Bifurcat Chaos, 2002, 12: 659-661

18 Chen G, Ueta T. Yet another chaotic attractor. Int J Bifurcat Chaos, 1999, 9: 1465-1466

19 Bao J H, Yang Q G. A new method to find homoclinic and heteroclinic orbits. Appl Math Comput, 2011, 217: 6526-6540

20 Zhou T, Tang Y, Chen G. Chen's attractor exists. Int J Bifurcat Chaos, 2004, 9: 3167-3177

21 Zhou L, Chen F. Sil'nikov chaos of the Liu system. Chaos, 2008, 18: 013113

22 Wang X, Li J, Fang J. Sil'nikov chaos of a 3-D quadratic autonomous system with a four-wing chaotic attractor. In: Poceedings of the 30th Chinese Conference, Yantai, China, 2011

23 Jiang Y, Sun J. Si'lnikov homoclinic orbits in a new chaotic system. Chaos Solit Fract, 2007, 32: 150-159

24 Zheng Z, Chen G. Existence of heteroclinic orbits of the Shil'nikov type in a 3D quadratic autonomous chaotic system. J Math Anal Appl, 2006, 315: 106-119

25 Bao J H, Yang Q G. Complex dynamics in the stretch-twist-fold flow. Nonlinear Dyn, 2010, 61: 773-781

26 Silva C P. Sil'nikov Theorem - A tutorial. IEEE Trans Circuits Syst I Fund Theory, 1993, 40: 675-682

Open Access This article is distributed under the terms of the Creative Commons Attribution License which permits any use, distribution, and reproduction in any medium, provided the original author(s) and source are credited. 